Provided for non-commercial research and education use. Not for reproduction, distribution or commercial use.

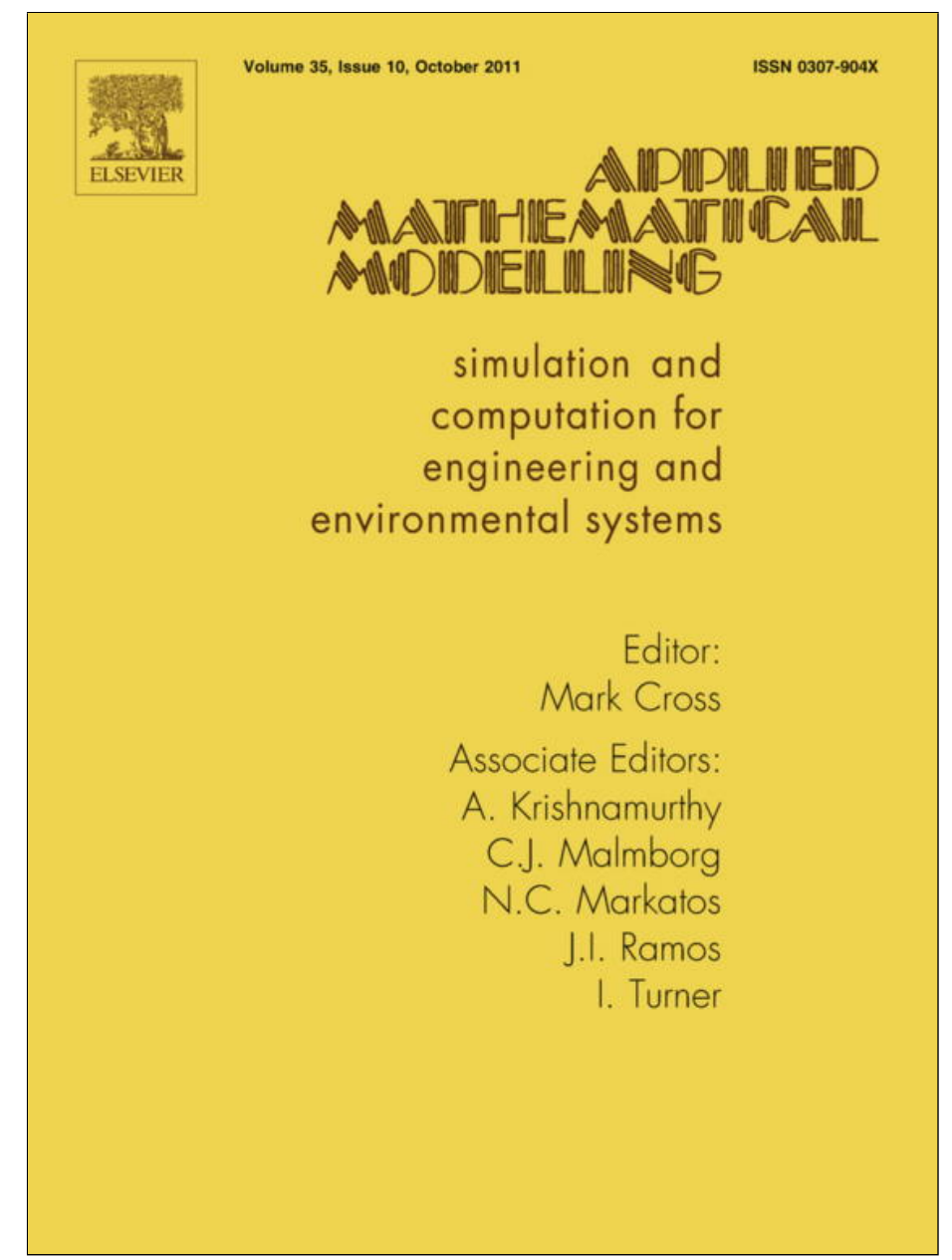

This article appeared in a journal published by Elsevier. The attached copy is furnished to the author for internal non-commercial research and education use, including for instruction at the authors institution and sharing with colleagues.

Other uses, including reproduction and distribution, or selling or licensing copies, or posting to personal, institutional or third party websites are prohibited.

In most cases authors are permitted to post their version of the article (e.g. in Word or Tex form) to their personal website or institutional repository. Authors requiring further information regarding Elsevier's archiving and manuscript policies are encouraged to visit:

http://www.elsevier.com/copyright 


\title{
Mathematical modelling of swirling flow in hydraulic turbines for the full operating range
}

\author{
R.F. Susan-Resiga ${ }^{\mathrm{a}, *}$, S. Muntean ${ }^{\mathrm{b}}$, F. Avellan ${ }^{\mathrm{c}}$, I. Anton ${ }^{\mathrm{a}}$ \\ a "Politehnica” University of Timişoara, Hydraulic Machinery Dpt., Bvd. Mihai Viteazu 1, RO-300222 Timişoara, Romania \\ ${ }^{\mathrm{b}}$ Romanian Academy - Timişoara Branch, Bvd. Mihai Viteazu 24, RO-300223, Timişoara, Romania \\ ${ }^{\mathrm{c}}$ École Polytechnique Fédérale de Lausanne, Laboratory for Hydraulic Machines, Av. de Cour 33Bis, CH-1007, Lausanne, Switzerland
}

\section{A R T I C L E I N F O}

\section{Article history:}

Received 29 September 2010

Received in revised form 16 March 2011

Accepted 31 March 2011

Available online 5 April 2011

\section{Keywords:}

Swirling flow

Hydraulic turbine

Constrained variational problem

Fourier-Bessel series

\begin{abstract}
A B S T R A C T
We introduce and validate a novel mathematical model for computing the radial profiles of both axial and circumferential velocity components, respectively, of the swirling flow exiting the runner of hydraulic turbines within the full operating range. We assume an incompressible, inviscid, axisymmetrical, and steady swirling flow, with vanishing radial velocity at runner outlet. First we find the correlation between the flux of moment of momentum downstream the turbine runner and the operating regime given by turbine's discharge and head. Second, we express the relationship between the axial and circumferential velocity components, corresponding to the fixed pitch runner blades, using the swirl-free velocity instead of the traditional relative flow angle at runner outlet. It is shown that the swirl-free velocity profile practically does not change with the operating regime. Third, we introduce a constrained variational problem corresponding to the minimization of the flow force while maintaining the prescribed discharge and flux of moment of momentum. This formulation also accounts for a possible central stagnant region to develop when operating the turbine far from the best efficiency point. Fourth, we show that by representing the unknown axial velocity profile with a suitable Fourier-Bessel series, the discharge constraint can be automatically satisfied. The resulting numerical algorithm is robust and produces results in good agreement with available data for both axial and circumferential velocity profiles measured on a model Francis turbine at several operating regimes. Our mathematical model is suitable for the early optimization stages of the runner design, as it provides the swirling flow configuration at runner outlet without actually computing the runner. By optimizing the parameterized swirl-free velocity profile one can achieve through the inverse design approaches the most suitable runner blades configuration at the trailing edge.
\end{abstract}

(c) 2011 Elsevier Inc. All rights reserved.

\section{Introduction}

Modern hydraulic turbines meet new challenges associated with the variable demand on the energy market as well as limited energy storage capabilities, resulting in great flexibility required in operation. Quite often turbines tend to be operated over an extended range of regimes far from the best efficiency point. In particular, Francis turbines, which have a fixed pitch runner, experience an abrupt decrease in efficiency and severe pressure fluctuations at off-design operating regimes. In

\footnotetext{
* Corresponding author. Tel.: +40 256403689; fax: +40 256403892.

E-mail addresses: resiga@mh.mec.upt.ro (R.F. Susan-Resiga), seby@acad-tim.tm.edu.ro (S. Muntean), francois.avellan@epfl.ch (F. Avellan), anton@ acad-tim.tm.edu.ro (I. Anton).
} 


\author{
Nomenclature \\ Notation definition \\ $Q\left[\mathrm{~m}^{3} / \mathrm{s}\right], \phi[-]$ volumetric turbine discharge and dimensionless discharge coefficient \\ $H[m], \psi[-]$ turbine head and dimensionless specific energy coefficient \\ $\eta[-] \quad$ hydraulic efficiency \\ $\rho\left[\mathrm{kg} / \mathrm{m}^{3}\right]$ liquid density \\ $\Omega[\mathrm{rad} / \mathrm{s}]$ runner angular speed \\ $R[m], r[-]$ radius, dimensional and dimensionless \\ $y[-] \quad$ dimensionless radius squared \\ $V[\mathrm{~m} / \mathrm{s}], v[-]$ velocity, dimensional and dimensionless \\ $P[\mathrm{~Pa}], p[-]$ pressure, dimensional and dimensionless \\ $\alpha[\mathrm{rad}], \beta[\mathrm{rad}]$ absolute and relative flow angles, respectively \\ $M\left[\mathrm{~kg} \mathrm{~m} \mathrm{~m}^{2} / \mathrm{s}^{3}\right], m[-]$ flux of moment of momentum, dimensional and dimensionless \\ Index definition \\ ref reference section at runner outlet \\ sf swirl-free \\ $z, r, \theta \quad$ axial, radial, and circumferential, respectively \\ w wall \\ s stagnation \\ $\star \quad$ reference operating regime \\ 1, 2 upstream/downstream the runner cross-sections
}

his review of confined vortices in flow machinery Escudier [1] noticed that the when hydraulic turbines operate at partial load a high level of residual swirl in the draft tube results from a mismatch between the swirl generated by the wicket gates (guidevanes) and the angular momentum extracted by the turbine runner. The vortex breakdown associated with this high level swirl, and in particular helical vortex breakdown, is seen as the source of flow unsteadiness and associated pressure fluctuations.

Evaluation of the turbine efficiency for the whole range of admissible discharge and head was, and still is, the standard experimental investigation on model turbines in order to predict the performances of the real size machine. The resulting efficiency "hill chart" usually displays a peek efficiency at the so-called "best efficiency point" (BEP). For the past decade, progress in computer algorithms, software and hardware made possible the numerical prediction of the hill-chart with reasonable accuracy, [2]. In addition, numerical investigation of the flow on the complete Francis turbine allows the analysis of the hydraulic losses on (i) spiral casing, (ii) guide vanes, (iii) runner, and (iv) draft tube, respectively. As shown by Vu and Retieb [2], the hydraulic losses in the first three segments of the turbine hydraulic passage have a rather modest variation with the operation regime. This is mainly the result of the significant progress in turbine design over the past decades. On the other hand, the draft tube which is the machine component where the flow exiting the runner is decelerated, thereby converting the excess of kinetic energy into static pressure, displays an abrupt increase in hydraulic losses as the operating regime departs from the BEP. Practically, for modern Francis turbines the shape of the hill-chart is dictated by the losses in the draft tube. As a result it is not surprising that a lot of research efforts are devoted now to the draft tube hydrodynamics.

The FLINDT (Flow Investigation in Draft Tubes) project [3] was aimed at extensive, state of the art, experimental investigations of both velocity and pressure fields in the draft tube of hydraulic turbines. In the case of machine rehabilitation of an existing power plant, mostly only the runner and the guide vanes are currently modified. For economical and safety reasons, the spiral casing and the draft tube are seldom redesigned. However, it is still a challenge to determine the optimum flow distribution at the runner outlet which leads to the best overall performance of the machine, including efficiency, power output and smooth regime for an extended operating range. The swirling flow at runner outlet measured in the first phase of the FLINDT project has been analyzed by Susan-Resiga et al. [4] who discovered that the axial and circumferential velocity profiles can be simultaneously represented as a superposition of three vortices: (i) a rigid body rotation with constant axial velocity, (ii) a counter-rotating Batchelor vortex with large characteristic radius, and (iii) a co-rotating Batchelor vortex with small characteristic radius. The last vortex is associated with the wake of the runner hub (also called crown), while the other two vortices are the direct outcome of the runner design. The stability analysis of this swirling flow [4] showed that the flow experiences a transition from supercritical to subcritical, according to the terminology introduced by Benjamin [5], as the discharge decreases, and this transition was shown to correspond to the discharge value where the pressure recovery in the draft tube suffers an abrupt change. When this phenomenon occurs in the neighborhood of the BEP, as it was the case for the Francis turbine investigated in the FLINDT project, obviously the machine operation is hindered. This sudden change in the draft tube performance as the turbine discharge is varied was also correlated by Mauri et al. [6] with the numerically 
found Werlé-Legendre flow separation on the draft tube wall. It was clear from these investigations that the swirling flow ingested by the draft tube is the key issue in both minimizing the draft tube losses and avoiding unexpected hydrodynamic effects, respectively. While successful in analyzing the swirling flow at runner outlet, our approach [4], further extended for the draft tube cone [7], lacks the capability of predicting the swirl configuration over a wide operating range. In particular, at low discharge conditions investigated in the second phase of the FLINDT project, where a precessing helical vortex is developed in the draft tube cone as a result of the self-induced swirl instability, also called helical vortex breakdown [8], with associated hydro-acoustic pressure waves [9], the three-vortex model from [4] fails to predict the circumferentiallyaveraged annular swirling flow with central quasi-stagnant region. However, we were able to compute this particular swirling flow in the draft tube cone [10], using axisymmetric turbulent swirling flow models, in good agreement with experimental data. We have used the Realizable $k-\epsilon$ turbulence model (RKE) in our axisymmetric flow simulations [10,7], but also the Reynolds-Stress model (RSM) or the renormalization group (RNG) $k-\epsilon$ model [11] are valid choices.

Predicting and understanding the swirling flow particularities at the runner outlet/draft tube inlet is also the key in developing novel flow control techniques aimed at mitigating the unwanted flow instabilities at partial discharge. Susan-Resiga et al. [12] propose a water jet injection from the runner crown, along the machine axis, and show that this method successfully mitigates the precessing helical vortex and the associated pressure fluctuations. This method was also confirmed by Zhang et al. [13] who also approximate the swirl in the draft tube cone with a Batchelor vortex in order to correlate the vortex rope occurrence with previous studies on the global instability of the swirling flow.

Most of the studies on draft tube hydrodynamics rely on velocity data at the draft tube inlet obtained either by direct measurements $[6,8]$ or by computing the flow in the turbine runner [14,15]. It is clear however that the swirling flow ingested by the draft tube is directly related to the runner blades geometry in the neighborhood of the trailing edge. The state of the art in Francis turbine design half a century ago is well exemplified by Bovet [16]. With respect to the flow at Francis runner outlet, Bovet mentioned that the designer's choice for the absolute circumferential velocity is practically arbitrary. Following the argument in [16] one should keep in mind that the kinetic energy associated with the circumferential velocity downstream the runner cannot be recovered in the turbine diffuser (draft tube) and as a result it should be considered as a hydraulic loss. It follows that the runner should be designed with vanishing flow rotation at outlet. However, it is recommended to leave a small flow rotation at runner outlet in order to improve the flow in the draft tube cone. Without inlet rotation, the decelerated flow in the conical diffuser downstream the runner may have large hydraulic losses due to the flow detachment at the cone wall. This is particularly true for modern compact discharge cones with rather large cone angle. The benefits of inlet swirl on pressure recovery in conical diffusers has been well documented by Fox et al. [17]. As a result, Bovet [16] recommended to leave a small circumferential velocity component when designing the runner, arguing that the losses associated with this excess in outlet kinetic energy is more than compensated by the improvement in the draft tube performance. In quantitative terms, Bovet introduced the somehow arbitrary rule of choosing the magnitude of the relative velocity $W$ equal to the transport velocity $U$ on a mid streamline. Once the discharge velocity component is given at the design operating point, the above condition allows the computation of absolute circumferential velocity from the velocity triangle. Other choices for the runner design are detailed by Anton [18, Ch. 8]. Since a rigorous theory for choosing and optimizing the swirling flow at Francis runner outlet is still lacking, we attempt such a development in this paper.

The modern approaches use fully three-dimensional inverse design methods for turbomachinery blades, [19]. In this method the distribution of the radius $\times$ circumferential velocity on the meridional geometry of the runner is prescribed and the corresponding blade shape is computed iteratively. For a Francis hydraulic turbine the design data upstream the runner are relatively easy to prescribe for the flow exiting the guide vanes. However, downstream the runner we still need a systematic approach to determine the best swirling flow configuration such that the turbine will have high efficiency and smooth operation over an extended range. The real challenge is to determine the best choice of the flow at runner outlet before actually designing and analyzing the runner. It is this challenge we address in the present paper.

If one attempts to use the large body of literature devoted to swirling flows and vortex breakdown phenomenon for hydraulic turbines design, the main difficulty is that most of the papers consider theoretical swirl configurations associated with a one parameter Batchelor vortex, [20], or two-parameter vortex [21]. Even if using such simple analytical vortex representations may be useful in analyzing and understanding measured [4] or computed [13] turbine swirling flows, it is clear that we need an approach to actually construct the swirling flow configurations encountered in hydraulic turbines at runner outlet, if possible without computing the runner flow.

The paper is organized as follows. In Section 2 we introduce a simple model for analyzing the turbine operation over the full intended range. The main result of this model is the relationship between the flux of moment of momentum at runner outlet as function of turbine's discharge and head. Section 3 analyzes the kinematic constrained imposed on the swirling flow by the fixed-pitch runner blades. In doing so, we introduce the swirl-free velocity profile and show that it has a simple shape, practically independent on the operating regime. The main result of the paper is given in Section 4, where we present the constrained variational problem for the swirling flow at runner outlet. The numerical approximation for the unknown axial velocity profile is developed in Section 5, where we show that a Fourier-Bessel series automatically preserves the turbine discharge. The numerical approach for solving the variational problem is underlined in Section 6, where we also present a set of numerical results and validation against experimental data for axial and circumferential velocity profiles. The agreement between our model predictions and experimental data, in the framework of model simplified assumptions, is discussed in Section 7. The paper conclusions are summarized in the last section. 


\section{Francis turbine operation at variable regimes}

The analysis of the hydraulic turbine operation at variable regimes starts with the fundamental equation of turbomachines, written for a hydraulic turbine as

$$
\eta(\rho Q)(g H)=\underbrace{\int_{S_{1}}\left(\Omega R V_{1 \theta}\right) \rho V_{1 r} \mathrm{~d} S_{1}}_{M_{1}}-\underbrace{\int_{S_{2}}\left(\Omega R V_{2 \theta}\right) \rho V_{2 z} \mathrm{~d} S_{2}}_{M_{2}} .
$$

In the left-hand side of Eq. (1) we have the hydraulic power written as the product of the mass flow rate $\rho Q$ and specific energy $g H$, multiplied by the hydraulic efficiency $\eta$. In the right-hand side of Eq. (1) we have the rate at which the fluid does work on the rotor, which by Newton's second law applied to the moment of forces is equal to the difference in the flux of moment of momentum between cross-sections upstream the runner, $S_{1}$, and downstream the runner, $S_{2}$, respectively. Figs. 1 and 2 show that $S_{1}$ is a cylindrical cross-section located at the outlet of the guide vanes, while $S_{2}$ is a plane normal to the machine axis located just downstream the runner outlet. Obviously, when computing the flux of moment of momentum upstream the runner, $M_{1}$, we use the discharge velocity $V_{1 r}$, while for $M_{2}$ the discharge velocity is $V_{2 z}$. The circumferential velocity uspstream the runner is $V_{1 \theta}$, while downstream the runner we have $V_{2 \theta}$.

The above equation can be written in dimensionless form by introducing the following coefficients:

$$
\begin{aligned}
\phi & \equiv \frac{\rho Q}{\rho\left(\Omega R_{\text {ref }}\right) \pi R_{\text {ref }}^{2}} \text { discharge coefficient, } \\
\psi & \equiv \frac{g H}{\left(\Omega R_{\text {ref }}\right)^{2} / 2} \text { energy coefficient, } \\
m & \equiv \frac{M}{\rho\left(\Omega R_{\text {ref }}\right)^{3} \pi R_{\text {ref }}^{2}} \quad \text { flux of moment of momentum coefficient. }
\end{aligned}
$$

The reference radius $R_{\text {ref }}$ is the runner outlet radius, as shown in Fig. 1. We can rewrite now Eq. (1) in the simple dimensionless form:

$$
\eta \phi \frac{\psi}{2}=m_{1}-m_{2}
$$

A turbine operating regime is defined by the turbine discharge and specific energy, i.e. by the pair $(\phi, \psi)$. For the preliminary design of a hydraulic turbine, the hydraulic efficiency can be considered from an estimated hill-chart $\eta(\phi, \psi)$. Our goal for the present investigation is to evaluate the flux of moment of momentum downstream the runner,

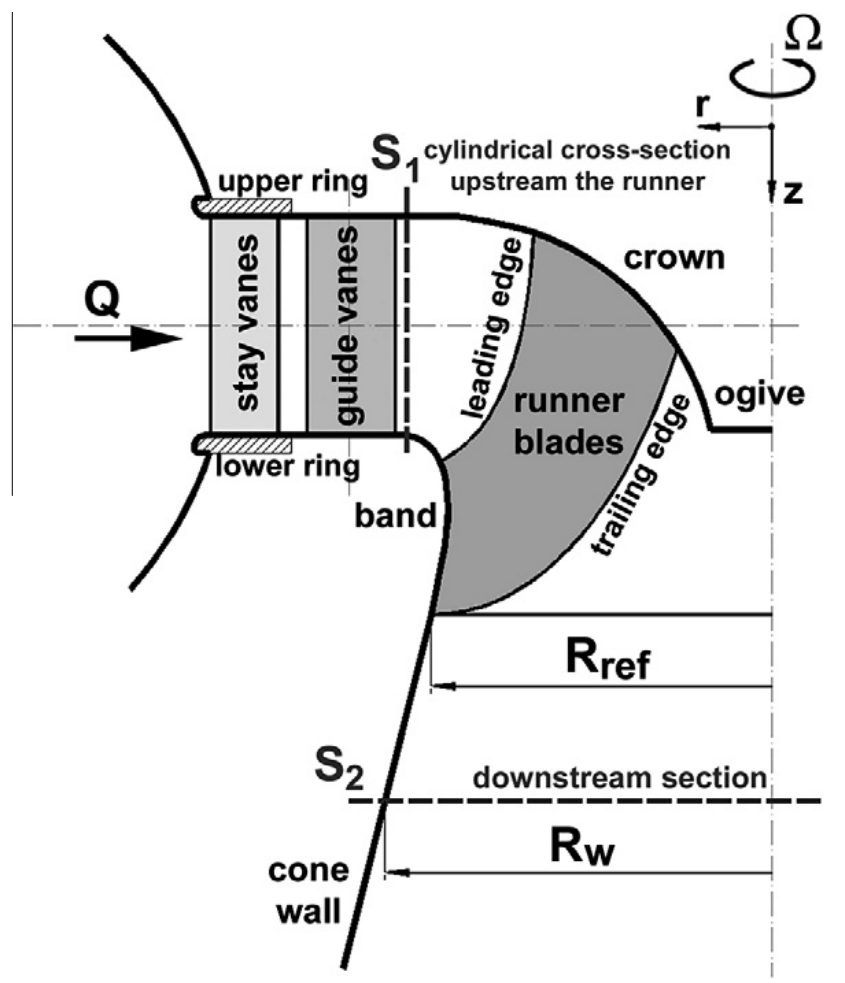

Fig. 1. Meridian cross-section of a Francis turbine. 


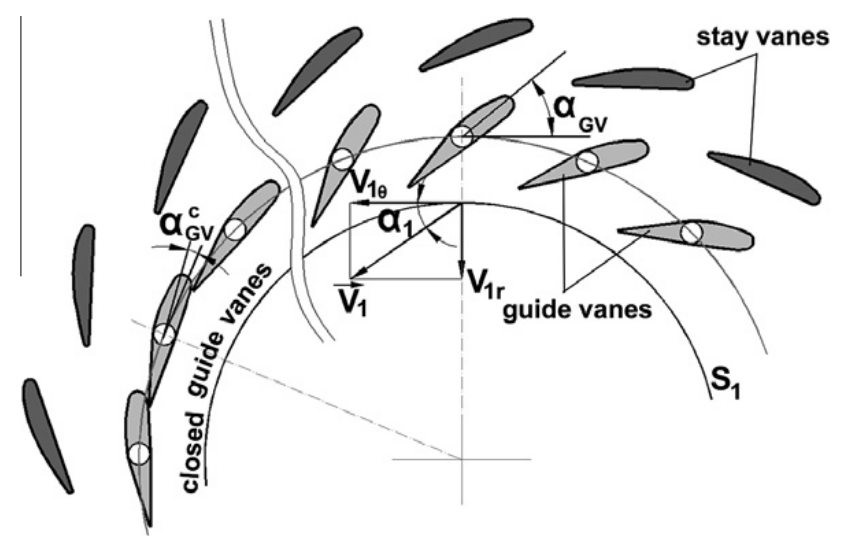

Fig. 2. Flow angle $\alpha_{1}$ at distributor outlet/runner inlet.

$$
m_{2}(\phi, \psi)=m_{1}(\phi, \psi)-\eta \phi \frac{\psi}{2}
$$

In order to use Eq. (4) we need to build an approximation for $m_{1}(\phi, \psi)$ taking into account that the turbine discharge is modified by opening or closing the guide vanes, Fig. 2.

In our simplified model we consider an averaged velocity at guide vanes outlet, with the magnitude $V_{1}$ and the flow angle $\alpha_{1}$ with respect to the tangential direction. Note that $\alpha_{1}$ is not the same as the guide vane opening angle $\alpha_{\mathrm{GV}}$, but a correlation can be found by solving for the flow in the two circular cascades corresponding the both stay vanes and guide vanes. The velocity magnitude should depend on the turbine head as $V_{1} \sim \sqrt{\eta g H}$. On the other hand, the discharge is proportional to the radial velocity component, $Q \sim V_{1 r}=V_{1} \sin \alpha_{1}$. The moment of momentum flux should be proportional to the product of radial and circumferential velocity components, $M_{1} \sim V_{1 r} V_{1 \theta}=V_{1}^{2} \sin \alpha_{1} \cos \alpha_{1}$. These considerations should be viewed as first approximations to be used for the preliminary design stages.

If we consider a reference operating point (e.g. the design operating point, or the best efficiency point for an existing runner), further denoted by $\left(\phi^{\star}, \psi^{\star}\right)$, the above considerations lead to

$$
\begin{aligned}
& \frac{\phi}{\phi^{\star}}=\sqrt{\frac{\eta \psi}{\eta^{\star} \psi^{\star}}} \frac{\sin \alpha_{1}}{\sin \alpha_{1}^{\star}}, \\
& \frac{m_{1}}{m_{1}^{\star}}=\frac{\eta \psi}{\eta^{\star} \psi^{\star}} \frac{\sin 2 \alpha_{1}}{\sin 2 \alpha_{1}^{\star}} .
\end{aligned}
$$

Given an arbitrary operating point $(\phi, \psi)$, Eqs. (5) form a system of two algebraic equations with the unknowns $m_{1}$ and $\alpha_{1}$. Note that at the reference operating point $\left(\phi^{\star}, \psi^{\star}\right)$ we assume $\eta^{\star}, \alpha_{1}^{\star}$, and $m_{1}^{\star}$ as known quantities. With $m_{1}$ computed by solving Eqs. (5) we can find from Eq. (4) the flux of moment of momentum downstream the runner at any operating point.

In order to validate this mathematical model we consider several operating regimes for a Francis model turbine, where both axial and circumferential velocity profiles have been measured in a survey section just downstream the runner, $S_{2}$ section in Fig. 1, using Laser Doppler Velocimetry, [4,10]. Using the experimental data for the axial and circumferential velocity components, shown in Section 6 of this paper, we have computed the turbine discharge and the flux of moment of momentum as follows:

$$
\begin{aligned}
& Q=\int_{0}^{R_{\mathrm{w}}} V_{2 z} 2 \pi R \mathrm{~d} R, \\
& M_{2}=\int_{0}^{R_{\mathrm{w}}}\left(\Omega R V_{2 \theta}\right) \rho V_{2 z} 2 \pi R \mathrm{~d} R,
\end{aligned}
$$

where $R_{\mathrm{w}}$ is the wall radius in section $S_{2}$.

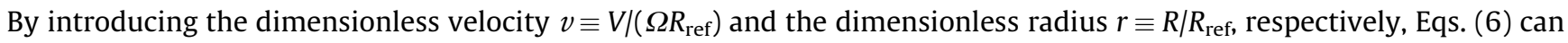
be written in dimensionless form using the definitions (2a) and (2c),

$$
\begin{aligned}
& \phi=\int_{0}^{r_{\mathrm{w}}} v_{2 z} 2 r \mathrm{~d} r \\
& m_{2}=\int_{0}^{r_{\mathrm{w}}}\left(r v_{2 \theta}\right) v_{2 z} 2 r \mathrm{~d} r .
\end{aligned}
$$

The integrals in (7a) and (7b) are computed for the data shown in Fig. 6 using a simple trapezoidal rule, and the corresponding values are given in Table 1. 
Table 1

Experimental data for operating regimes with variable discharge $\phi$ and constant specific energy $\psi=1.18$.

\begin{tabular}{lll}
\hline $\begin{array}{l}\text { Relative turbine } \\
\text { discharge }\end{array}$ & $\begin{array}{l}\text { Discharge coefficient } \\
\phi\end{array}$ & $\begin{array}{l}\text { Moment of momentum flux coefficient } \\
m_{2}\end{array}$ \\
\hline$Q=0.714 Q_{\text {BEP }}$ & 0.26428 & 0.048341 \\
$Q=0.919 Q_{\text {BEP }}$ & 0.34015 & 0.036829 \\
$Q=0.974 Q_{\text {BEP }}$ & 0.36066 & 0.030264 \\
$Q=Q_{\text {BEP }}$ & 0.37014 & 0.028227 \\
$Q=1.025 Q_{\text {BEP }}$ & 0.37950 & 0.025154 \\
$Q=1.050 Q_{\text {BEP }}$ & 0.38881 & 0.021944 \\
$Q=1.107 Q_{\text {BEP }}$ & 0.40976 & 0.013239 \\
\hline
\end{tabular}

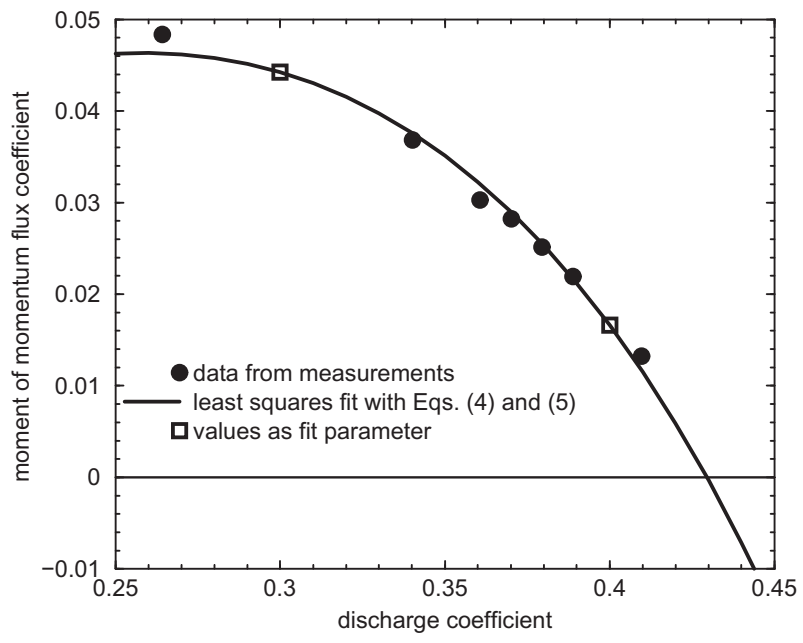

Fig. 3. Dimensionless flux of moment of momentum downstream the runner, $m_{2}$, versus the discharge coefficient, $\phi$.

Six of the operating points in Table 1 are clustered within $\pm 10 \%$ the discharge at the best efficiency point, while the point at $0.7 Q_{\text {BEP }}$ corresponds to the partial discharge regime with well-defined precessing vortex rope. The data from Table 1 are shown with filled circles in Fig. 3, where the solid line corresponds to the least squares fit using Eqs. (4) and (5). All data points correspond to the constant energy coefficient $\psi^{\star}=1.18$. The reference discharge coefficient $\phi^{\star}$ can be arbitrary chosen and it does not have to coincide with a data point. For example, by choosing $\phi^{\star}=0.3$ the fit parameters obtained with the IMSL [22] routine RNLIN are $m_{2}^{\star}=0.044232$ and $\alpha_{1}^{\star}=30.348^{\circ}$, respectively. When choosing $\phi^{\star}=0.4$ the fit parameters are $m_{2}^{\star}=0.016612$ and $\alpha_{1}^{\star}=42.351^{\circ}$, respectively. However, the fit curve is unchanged with the two points $m_{2}^{\star}\left(\phi^{\star}\right)$ on the curve, as shown in Fig. 3 with squares. We conclude that the least squares fit procedure based on Eqs. (4) and (5) is robust in the sense that the fit curve does not depend, as expected, on the choice of the reference discharge $\phi^{\star}$.

One can see that the simple mathematical model introduced in this section is in quite good agreement with the experimental data. No data were available to check the model for variable head, but it is clear that the model captures correctly the qualitative behavior of the residual flux of moment of momentum downstream the runner as the turbine is operated within a wide range. Moreover, this model is not intended for analyzing the turbine swirling flow. Instead, our model provides valuable information in the early design and optimization stages for Francis runners. In particular, the model provides the two main integral quantities defining the swirling flow downstream the runner, i.e. the discharge $\phi$ and the flux of moment of momentum $m_{2}$, respectively, for the whole intended operating range of the turbine.

\section{Swirling flow kinematics at Francis runner outlet}

For turbine runners with fixed pitch blades, such as Francis runners, the swirling flow at the runner outlet must satisfy the kinematic constraints given by the runner blades geometry close to the trailing edge.

The well-known velocity triangle, shown in Fig. 4, provides the kinematic constraint corresponding to the relative flow angle $\beta_{2}$. Note that $\beta_{2}$ is not the blade angle at the trailing edge. Moreover, $\beta_{2}$ is changing along the radius, corresponding to the blade geometry from hub to shroud. For the present simplified model we assume that $\beta_{2}$ depends only on the radius for the section $S_{2}$ at runner outlet. In doing so, we consider that this $\beta_{2}$ radial variation is not changing with the operating point. This assumption is acceptable for Francis turbines, where the large number of blades provide a good guidance of the flow with minimal three-dimensional effects in the inter-blade channel. However, it is clear that the relative flow angle $\beta_{2}$ 


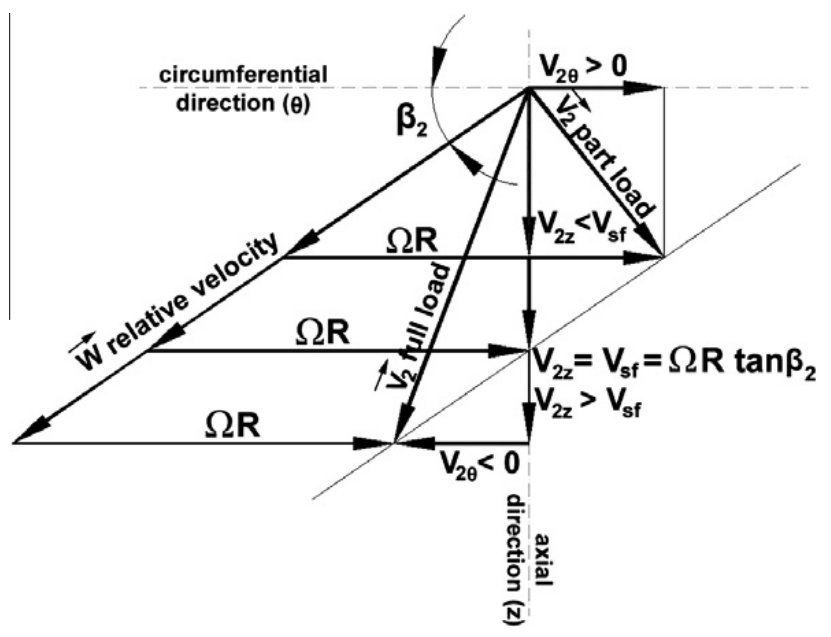

Fig. 4. Velocity triangle downstream the runner, for variable operating regimes.

changes with the operating point even for inviscid flows, but here we assume that such variations could be neglected in a first approximation.

Fig. 4 shows three particular configurations of the velocity triangle, with the relative velocity kept on the same direction given by $\beta_{2}$. Also, the transport velocity $\Omega R$ remains the same for all three cases further examined. At low discharge, corresponding to turbine's part-load regimes, the absolute circumferential velocity $V_{2 \theta}$ has the same direction as the transport velocity, and the swirling flow co-rotates with respect to the runner. At large discharge, corresponding to turbine's full-load operating regimes, $V_{2 \theta}$ is in opposite direction with the transport velocity, and the swirl counter-rotates with respect to the runner. In-between we can always identify a regime where the absolute circumferential velocity vanishes, i.e. $V_{2 \theta}=0$. We call the corresponding axial velocity the "swirl-free velocity", $V_{\mathrm{sf}}$. Since the relative flow angle is directly related to the swirl-free velocity as $\tan \beta_{2}=V_{\mathrm{sf}} /(\Omega R)$, we can use the $V_{\mathrm{sf}}$ instead of $\beta_{2}$ to describe the kinematical flow constraint at runner outlet. For an arbitrary operating regime we have,

$$
\frac{V_{2 z}}{\Omega R-V_{2 \theta}}=\tan \beta_{2}=\frac{V_{\text {sf }}}{\Omega R}
$$

thus the swirl free-velocity can be written as

$$
V_{\mathrm{sf}}=\frac{\Omega R V_{2 z}}{\Omega R-V_{2 \theta}}, \quad \text { or in dimensionless form } \quad v_{\mathrm{sf}}=\frac{r v_{2 z}}{r-v_{2 \theta}},
$$

with dimensionless radius and velocity defined as $r \equiv R / R_{\text {ref }}$ and $v \equiv V /\left(\Omega R_{\text {ref }}\right)$, respectively. Once the swirl-free velocity profile is known, $v_{\mathrm{sf}}(r)$, the circumferential velocity follows from the axial velocity profile as

$$
v_{2 \theta}=r\left(1-\frac{v_{2 z}}{v_{\mathrm{sf}}}\right)
$$

Eq. (10) shows that at part load, i.e. $v_{2 z}<v_{\mathrm{sf}}$, we have $v_{2 \theta}>0$ with co-rotating swirl, while at full-load, $v_{2 z}>v_{\mathrm{sf}}$, we have counter-rotating swirl with $v_{2 \theta}<0$.

The concept of swirl-free velocity has been employed by Kubota et al. [23] who investigate the draft tube losses for the GAMM model Francis turbine, and in particular the influence of the swirl intensity of runner outflow for variable operating points given by the discharge and specific energy coefficients. When analyzing the swirl velocity at runner outlet, they assume a constant runner outflow angle and express a representative circumferential velocity $V_{2 \theta}$ using the transport velocity $U_{2}=\Omega R$, the swirl-free discharge coefficient $\phi_{\mathrm{sf}}$, and the discharge coefficient value $\phi$ corresponding to the operating point, as $V_{2 \theta}=U_{2}\left(1-\phi / \phi_{\text {sf }}\right)$. Both $V_{2 \theta}$ and $U_{2}$ are considered by Kubota et al. [23] on a representative mid-streamline at runner outlet, corresponding to a radius of $0.1394 \mathrm{~m}$ while the runner outlet radius is $0.200 \mathrm{~m}$. This rather crude evaluation does not account for the axial and circumferential velocity profiles, and obviously it does not describe the evolution of the swirling flow configuration when changing the operating point. However, it introduces the idea of the swirl-free meridian velocity to replace the relative flow angle.

In this paper we develop the swirl-free concept, but instead of using a single value corresponding to an arbitrary chosen streamline we introduce a swirl-free velocity profile at runner outlet. Moreover, we argue that this is an essential ingredient for the runner design and optimization within a range of operating regimes.

The main hypothesis concerning the swirl-free velocity profile introduced above as an alternative to the relative flow angle at runner outlet is that $v_{\mathrm{sf}}(r)$ is practically unchanged as the operating regime spans the whole operating range of the turbine. Fig. 5 shows with filled circles the $v_{\text {sf }}$ values computed with Eq. (9) from the experimental data for axial and circumferential velocities measured at the operating points indicated in Table 1 . It can be seen that the experimental points are 


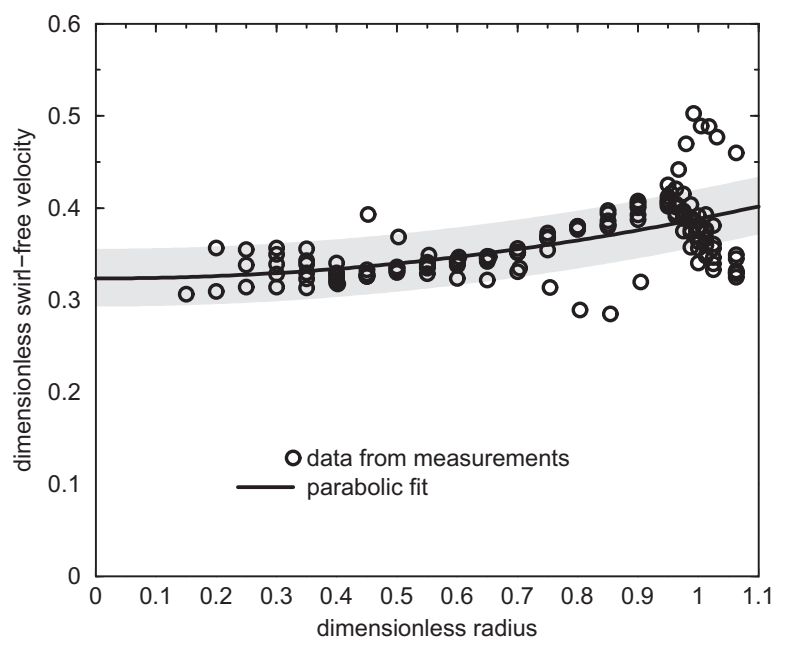

Fig. 5. The swirl-free velocity profile at a Francis runner model outlet.

reasonably clustered around the parabolic fit $v_{\mathrm{sf}}(r)=0.323505+0.0646465 r^{2}$, with a standard deviation for the errors of 0.03136 as shown with the grey strip in Fig. 5. We postulate that an expression of the form $v_{\mathrm{sf}}(r)=a+b r^{2}$ can be employed for optimizing the swirling flow downstream the Francis runner, with $a$ and $b$ as optimization parameters, as an alternative to consider the relative flow angle $\beta_{2}$ at runner outlet. The optimization of $v_{\mathrm{sf}}(r)$ should be performed mainly to minimize the weighted hydraulic losses in the turbine draft tube, or at least with respect to the losses in the draft tube cone.

\section{Variational formulation for swirling flow at Francis runner outlet}

In Section 2 we have shown that the swirling flow downstream the Francis turbine runner is characterized by two integral quantities: the discharge coefficient and the flux of moment of momentum coefficient. In addition, this swirling flow satisfies a kinematic constraint corresponding to the runner blade geometry, described in Section 3 by the swirl-free velocity profile. The swirling flow exiting the runner is further decelerated by the draft tube in order to convert the excess of kinetic energy into static pressure, thus reducing the overall losses in the hydraulic turbine. However, in order to evaluate the hydraulic losses in the draft tube, generated by both swirl deceleration and change in the overall flow direction one needs to know the axial and circumferential velocity profile at the draft tube inlet for the whole operating range of the turbine.

From now on, for the rest of the paper we will refer only to the swirling flow at the runner outlet $S_{2}$, thus we will drop the index 2 in order to simplify the notations.

In order to find the swirling flow configuration we will consider a simplified swirling flow model corresponding to steady, axisymmetric, incompressible and inviscid swirling flow. In this case, the Euler equations reduce to the Bragg-Hawthorne equation [24] for the stream-function. The symmetry investigations of the Bragg-Hawthorne equation led Frewer et al. [25] to the conclusion that it possesses additional symmetries not being admitted by the original Euler equations. As a result, it appears that not the Euler equations but rather a set of integro-differential equations attains full equivalence to the BraggHawthorne equation. A particularity of the Bragg-Hawthorne equation is that it includes two arbitrary functions, circulation and total specific energy, which depends only on the stream function by Kelvin's and Bernoulli's theorems, respectively. For the problem examined in this paper, the information available for the swirling flow downstream the Francis turbine runner does not allow the determination of the two generating functions in the Bragg-Hawthorne equation.

The Bragg-Hawthorne equation became the mathematical foundation for the throughflow theory of turbomachines. However, Oates et al. [26] introduced a variational formulation of the throughflow problem, leading to robust finite element algorithms. Their variational functional corresponds to the integral of the meridional momentum over all computational domain. The variational approach had been previously used by Benjamin [5] to explain the vortex breakdown phenomenon, where the functional was named "flow force". The variational formulation has been extended by Keller et al. [27] to allow variable lower endpoints, i.e. for cases when the flow occupies an annulus starting from a positive radius and going up to the wall. This approach has been further developed by Wang and Rusak [20] who explore both local and global minimizers of the variational functional for inviscid, incompressible and steady axisymmetric swirling flows to explain the dynamics of swirling flows in a pipe and transition to axisymmetric vortex breakdown.

Going back to the swirling flow at the Francis runner outlet, we consider that the flow is locally parallel (so-called columnar flow) due to the proximity to the turbine throat. Indeed, the radial velocity component close to the runner outlet is much smaller than either axial and circumferential velocities. Cervantes and Gustavsson [28] attempt to estimate the radial velocity in swirling flow at the draft tube inlet of a Kaplan turbine using the Bragg-Hawthorne equation (also known in literature as Long (1953)-Squire (1956) equation). However, we consider vanishing radial velocity at Francis runner outlet for the present simplified model. This assumption is also supported by experimental data of Tridon et al. [29]. 
The variational formulation for incompressible, inviscid, steady, columnar, swirling flow requires the minimization of the integral flow force,

$$
F \equiv \int_{0}^{R_{\mathrm{w}}}\left(\rho V_{z}^{2}+P_{\mathrm{w}}-P\right) 2 \pi R \mathrm{~d} R
$$

where we have employed the pressure deficit with respect to the wall pressure, $P_{\mathrm{w}}-P$ instead of the static pressure $P$. The corresponding dimensionless flow force and pressure are defined as

$$
\begin{aligned}
f & \equiv \frac{F}{\rho\left(\Omega R_{\mathrm{ref}}\right)^{2} \pi R_{\mathrm{ref}}^{2}}, \\
p & \equiv \frac{P_{\mathrm{w}}-P}{\rho\left(\Omega R_{\mathrm{ref}}\right)^{2}} .
\end{aligned}
$$

The dimensionless flow force can now be written as

$$
f=\int_{0}^{y_{\mathrm{w}}}\left(v_{z}^{2}+p\right) \mathrm{d} y
$$

where $y \equiv r^{2}$ being a modified radial coordinate.

The radial distribution of the pressure is correlated with the circumferential velocity through the radial equilibrium equation, i.e. the radial component of the axisymmetric Euler equation for vanishing radial velocity,

$$
\frac{1}{\rho} \frac{\mathrm{d} P}{\mathrm{~d} R}=\frac{V_{\theta}^{2}}{R}
$$

After integrating Eq. (14) and using Eq. (10) we obtain the dimensionless pressure,

$$
p(y)=\frac{1}{2} \int_{y}^{y_{\mathrm{w}}}\left(1-\frac{v_{z}}{v_{\mathrm{sf}}}\right)^{2} \mathrm{~d} x
$$

Given the swirl-free velocity profile, for example $v_{\mathrm{sf}}(y)=a+$ by as shown in Section 3 , the functional $f$ will depend only on the axial velocity profile $v_{z}(y)$ as follows:

$$
f\left(v_{z}\right)=\int_{0}^{y_{\mathrm{w}}} v_{z}^{2} \mathrm{~d} y+\frac{1}{2} \int_{0}^{y} \int_{y}^{y_{\mathrm{w}}}\left(1-\frac{v_{z}}{v_{\mathrm{sf}}}\right)^{2} \mathrm{~d} x \mathrm{~d} y .
$$

The minimization of functional $f\left(v_{z}\right)$ should be done with the integral constraints Eqs. (7), rewritten here as

$$
\begin{aligned}
& \int_{0}^{y_{\mathrm{w}}} v_{z} \mathrm{~d} y=\phi \\
& \int_{0}^{y_{\mathrm{w}}}\left(1-\frac{v_{z}}{v_{\mathrm{sf}}}\right) v_{z} y \mathrm{~d} y=m,
\end{aligned}
$$

where the values of $\phi$ and $m$ depend on the operating regime as shown in Section 2 . Once the axial velocity $v_{z}$ is found by minimizing (16) subject to constraints (17) and (18), the circumferential velocity follows from Eq. (10).

When the turbine operates at low discharge, the swirling flow downstream the runner develops a self-induced instability leading to an unsteady precessing helical vortex, also known as vortex rope. A qualitative model of this particular flow-field was given by Nishi et al. [30] who observed a quasi-stagnant (stalled) central region with the spiral vortex core wrapped around it. Of course, the flow in the central region is highly fluctuating, but on average all velocity components vanish. The thin shear layer between the central stalled region and the main annular swirling flow becomes unstable, breaks-up and rolls-up in a helical vortex with precession. This model has been further confirmed by the measurements of axial and circumferential velocity profiles made in Francis turbines [8,31], numerical simulations [10], or analytical models [32]. In order to incorporate the stagnant region model into the present variational formulation we proceed as Keller et al. [27] and introduce an additional unknown in the problem, $y_{s} \equiv r_{s}^{2}$, where $r_{s}$ is the radius of the central stagnant region. As a result, we re-formulate the variational problem corresponding to the swirling flow downstream the Francis turbine runner as follows:

Given the discharge coefficient, $\phi$, the dimensionless flux of moment of momentum, $m$, the swirl-free velocity profile $v_{s f}(y)$ and the radial extent of the cross-section at runner outlet, $y_{w}$, find the axial velocity profile $v_{z}(y)$ and the stagnant region extent $y_{s}$ that minimize the functional,

$$
f\left(v_{z}, y_{s}\right)=\int_{y_{\mathrm{s}}}^{y_{\mathrm{w}}} v_{z}^{2} \mathrm{~d} y+\frac{1}{2} \int_{y_{\mathrm{s}}}^{y_{\mathrm{w}}} \int_{y}^{y_{\mathrm{w}}}\left(1-\frac{v_{z}}{v_{\mathrm{sf}}}\right)^{2} \mathrm{~d} x \mathrm{~d} y,
$$

subject to the integral constraints, 


$$
\begin{aligned}
& \int_{y_{\mathrm{s}}}^{y_{\mathrm{w}}} v_{z} \mathrm{~d} y=\phi, \\
& \int_{y_{\mathrm{s}}}^{y_{\mathrm{w}}}\left(1-\frac{v_{z}}{v_{\mathrm{sf}}}\right) v_{z} y \mathrm{~d} y=m .
\end{aligned}
$$

The problem (19)-(21) admits solutions with jump in $v_{z}$ at $y_{s}>0$, in contrast to the solutions with central stagnant region obtained from Bragg-Hawthorne equation where $v_{z}\left(y_{s}\right)=0$, [27]. This is consistent with the circumferentially averaged velocity field from the analytical solution for the helical vortex in a pipe of Alekseenko et al. [32].

\section{Fourier-Bessel approximation of the axial velocity profile}

In order to solve constrained variational problem (19)-(21) we consider a representation of the axial velocity profile at runner outlet, $v_{z}(r)$, in terms of a truncated Fourier-Bessel series, [33]

$$
v_{z}(r)=v_{0}+\sum_{i=1}^{N} v_{i} J_{0}\left(\lambda_{i} \frac{r}{r_{\mathrm{w}}}\right)
$$

where $N$ is the finite number of modes and $\lambda_{i}$ are the zeros of the $J_{1}$ Bessel function, i.e. the non-zero solutions of $J_{1}\left(\lambda_{i}\right)=0$. These zeros are computed numerically and have the values $\lambda_{1}=3.831705970, \lambda_{2}=7.015586670, \lambda_{3}=10.17346814, \ldots$, with the asymptotic sequence $\lambda_{n}=(n+1 / 4) \pi+\mathcal{O}(1 / n)$. The Bessel functions in (22) are orthogonal,

$$
\int_{0}^{r_{\mathrm{w}}} J_{0}\left(\lambda_{i} \frac{r}{r_{\mathrm{w}}}\right) J_{0}\left(\lambda_{j} \frac{r}{r_{\mathrm{w}}}\right) r \mathrm{~d} r= \begin{cases}0 & \text { if } i \neq j \\ \frac{r_{\mathrm{w}}^{2}}{2} J_{0}^{2}\left(\lambda_{i}\right) & \text { if } i=j\end{cases}
$$

If we evaluate the discharge coefficient using Eq. (7a), we obtain

$$
\phi=\int_{0}^{r_{\mathrm{w}}} v_{0} 2 r \mathrm{~d} r+\sum_{i=1}^{N} v_{i} \int_{0}^{r_{\mathrm{w}}} J_{0}\left(\lambda_{i} \frac{r}{r_{\mathrm{w}}}\right) 2 r \mathrm{~d} r=v_{0} r_{\mathrm{w}}^{2}
$$

This result follows from the Bessel function property that

$$
\int_{0}^{r_{\mathrm{w}}} J_{0}\left(\lambda_{i} \frac{r}{r_{\mathrm{w}}}\right) r \mathrm{~d} r=0, \quad i=1,2, \ldots
$$

In other words, each mode $J_{0}\left(\lambda_{i} r / r_{\mathrm{w}}\right)$ has a vanishing contribution to the discharge. As a result, by replacing $v_{0}$ with $\phi / r_{\mathrm{w}}^{2}$ the Fourier-Bessel approximation of the axial velocity will always satisfy the discharge constraint (20).

Since we have introduced the modified radial coordinate $y=r^{2}$, we can re-write the discharge preserving axial velocity profile as

$$
v_{z}(y)=\frac{\phi}{y_{\mathrm{w}}}+\sum_{i=1}^{N} v_{i} J_{0}\left(\lambda_{i} \sqrt{\frac{y}{y_{\mathrm{w}}}}\right), \quad 0 \leqslant y \leqslant y_{\mathrm{w}} .
$$

When a stagnant region is present, i.e. $y_{\mathrm{s}} \leqslant y \leqslant y_{\mathrm{w}}$, with $y_{\mathrm{s}}>0$, each mode will have a non-zero contribution to the overall discharge as follows:

$$
\int_{y_{\mathrm{s}}}^{y_{\mathrm{w}}} J_{0}\left(\lambda_{i} \sqrt{\frac{y}{y_{\mathrm{w}}}}\right) \mathrm{d} y=-2 \frac{\sqrt{y_{\mathrm{s}} y_{\mathrm{w}}}}{\lambda_{i}} J_{1}\left(\lambda_{i} \sqrt{\frac{y_{\mathrm{s}}}{y_{\mathrm{w}}}}\right), \quad i=1,2, \ldots
$$

Using Eq. (27) we can compute the discharge for the annular section $y_{\mathrm{s}} \leqslant y \leqslant y_{\mathrm{w}}$ as

$$
\phi=\int_{y_{\mathrm{s}}}^{y_{\mathrm{w}}} v_{z}(y) \mathrm{d} y=v_{0}\left(y_{\mathrm{w}}-y_{\mathrm{s}}\right)-2 \sqrt{y_{\mathrm{s}} y_{\mathrm{w}}} \sum_{i=1}^{N} \frac{v_{i}}{\lambda_{i}} J_{1}\left(\lambda_{i} \sqrt{\frac{y_{\mathrm{s}}}{y_{\mathrm{w}}}}\right) .
$$

We can now write the discharge-preserving axial velocity representation as

$$
v_{z}(y)=\frac{\phi}{y_{\mathrm{w}}-y_{\mathrm{s}}}+\frac{2 \sqrt{y_{\mathrm{s}} y_{\mathrm{w}}}}{y_{\mathrm{w}}-y_{\mathrm{s}}} \sum_{i=1}^{N} \frac{v_{i}}{\lambda_{i}} J_{1}\left(\lambda_{i} \sqrt{\frac{y_{\mathrm{s}}}{y_{\mathrm{w}}}}\right)+\sum_{i=1}^{N} v_{i} J_{0}\left(\lambda_{i} \sqrt{\frac{y}{y_{\mathrm{w}}}}\right), \quad y_{\mathrm{s}} \leqslant y \leqslant y_{\mathrm{w}}
$$

Note that in this case the average discharge velocity $\phi /\left(y_{\mathrm{w}}-y_{\mathrm{s}}\right)$ should be corrected because the modes have no longer vanishing contributions to the overall discharge. Obviously, if $y_{s}$ vanishes in Eq. (29) we recover Eq. (26). 


\section{Numerical method and results}

With the Fourier-Bessel representation of the axial velocity profile, Eq. (29), the discharge constraint (20) is automatically satisfied and we have to minimize the functional (19), subject to the constraint (21), with respect to the unknowns $y_{\mathrm{s}}$ and $v_{i}$,

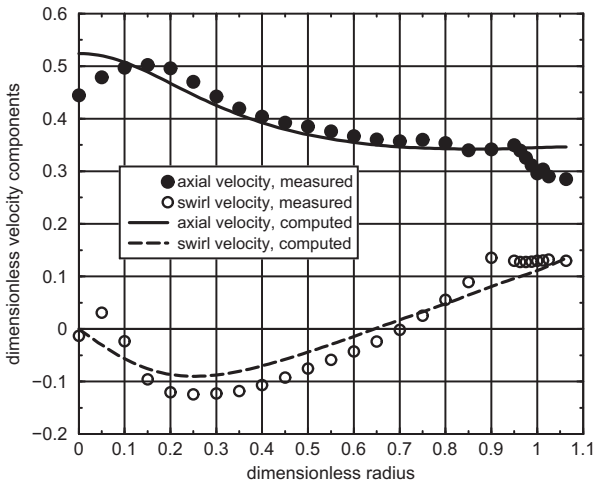

(a) $Q=1.107 Q_{\mathrm{BEP}}$

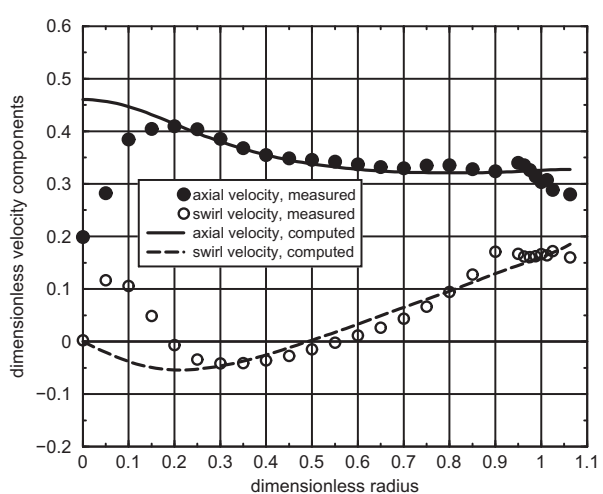

(c) $Q=1.025 Q_{\mathrm{BEP}}$

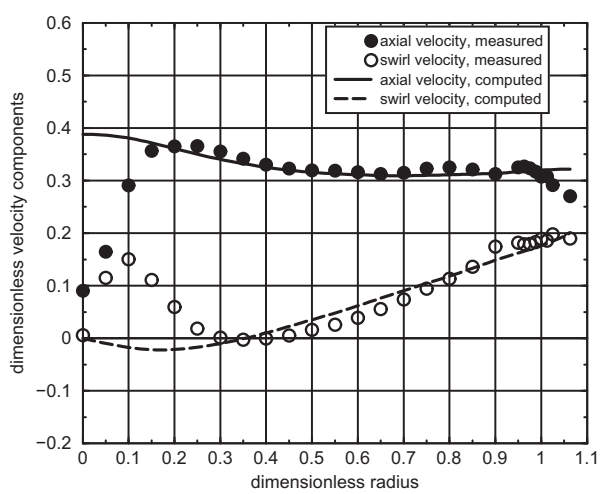

(e) $Q=0.974 Q_{\mathrm{BEP}}$

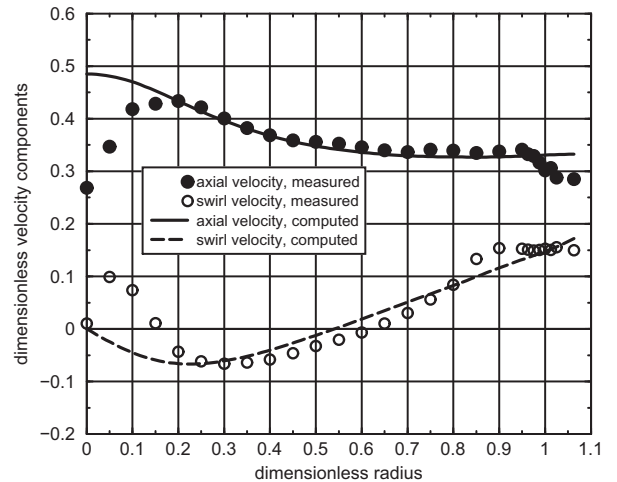

(b) $Q=1.050 Q_{\mathrm{BEP}}$

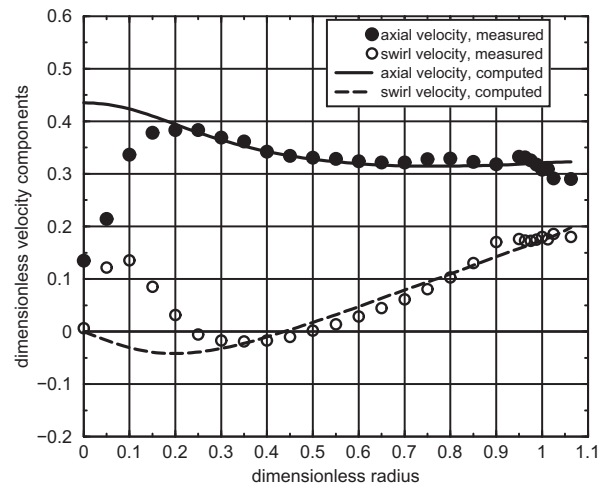

(d) $Q=Q_{\mathrm{BEP}}$

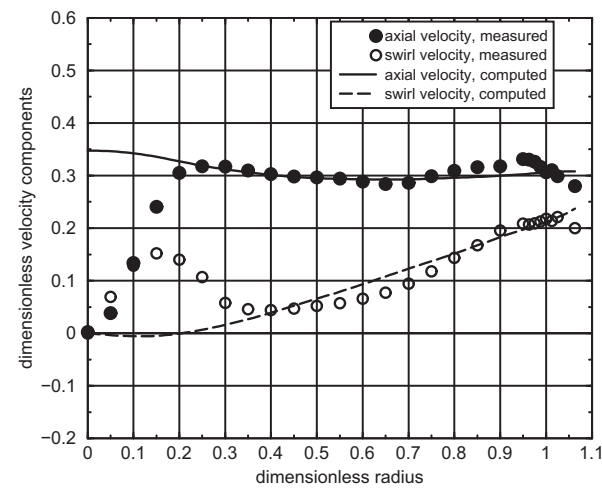

(f) $Q=0.919 Q_{\mathrm{BEP}}$

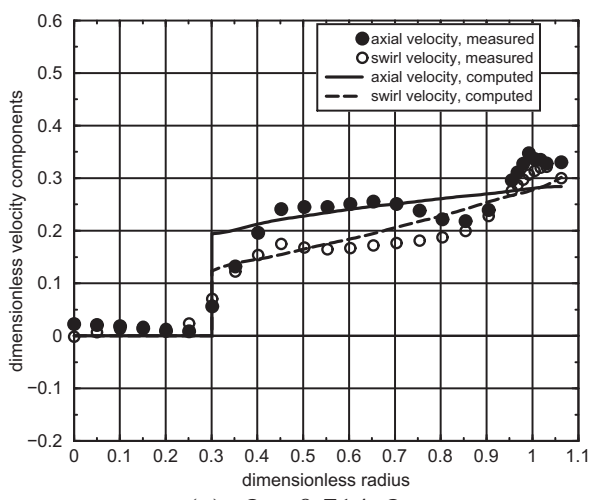

(g) $Q=0.714 Q_{\mathrm{BEP}}$

Fig. 6. Axial and circumferential velocity profiles at $S_{2}$ cross-section, Fig. 1 . 
$i=1,2, \ldots N$. For the numerical results further presented in this section we have used $N=9$, since the solution does not change anymore by adding Fourier-Bessel modes.

We have developed a computer code using the IMSL (International Mathematics and Statistics Library) FORTRAN numerical library, [22]. The constrained minimization problem (19), (21) is solved numerically with the IMSL routine NCONF which is based on subroutine NLPQL developed by Schittkowski [34]. It uses a successive quadratic programming method to solve the general nonlinear programming problem. The simple integrals are evaluated numerically using the QDAG subroutine which uses a globally adaptive scheme based on Gauss-Konrod rules, [35]. The two-dimensional integral in the functional (19) is computed with the TWODQ subroutine by iterated calls to QDAG. The Bessel functions are evaluated with BSJ0 and BSJ1, respectively. The zeros of the $J_{1}$ function are computed only once using Múller [36] method implemented by the ZREAL subroutine, with the initial guess $\lambda_{n}=(n+1 / 4) \pi$.

The numerical results presented in this section are aimed at validating the mathematical model introduced in the previous sections. As a result, we examine the operating points presented in Table 1, with the swirl-free velocity profile $v_{\mathrm{sf}}(y)=0.323505+0.0646465 y$ from Fig. 5 .

Fig. 6 shows the measured and computed velocity profiles, for axial and circumferential velocity components, respectively. The experimental data were obtained within the FLINDT project phase 1 [3], Figs. 6(a)-(f), and phase 2 [8], Fig. 6(g), respectively. The velocity was measured with a two-component probe Laser Doppler Anemometry (LDA), using back-scattered light. The measuring point geometrical location is controlled within a $0.05 \mathrm{~mm}$ accuracy, and uncertainties in velocity measurements are estimated at $2 \%$ of the measured value [37]. The same data have also been used in our previous investigations $[4,10,7]$. The computed axial velocity profile is shown with solid lines in Fig. 6 , and the circumferential velocity computed with Eq. (10) is represented with dashed lines. The dimensionless wall radius at $S_{2}$ is $r_{\mathrm{w}}=1.063$.

\section{Discussion}

Fig. 6 shows that our mathematical model correctly captures the swirling flow evolution as the turbine discharge is varied. At full load, $Q>Q_{B E P}$, one can see an increase of the axial velocity excess near the axis. On the other hand, at part load $Q<Q_{\mathrm{BEP}}$ this velocity excess is gradually decreased until a stagnant region develops in the axis neighborhood.

The agreement with experimental data within the $\pm 10 \%$ the best efficiency discharge $Q_{\mathrm{BEP}}$, Figs. 6 (a)-(f), is quite good except the wake of the crown which widens as the discharge decreases. This discrepancy is expected since our model does not account for the viscous effects which lead to the crown wake.

At low discharge, our model correctly captures the central stagnant region extent, Fig. 6(g), as well as the overall shape of the velocity components radial variation. The measured flow acceleration close to the wall is associated with the inter-blade vortex developed at the junction of the blade with the band [15, section 7.1]. Obviously, the present axisymmetric inviscid swirling flow model cannot capture the effects induced by such three-dimensional flow structures. The transition between the central stagnant region and the main annular swirling flow corresponds to the vortex sheet which evolves in the precessing helical vortex, [10]. In reality, the helical vortex has a finite core size and the corresponding circumferentially averaged flow displays a finite gradient in the stagnation - main flow transition zone, [38]. The swirl predicted by our simplified model corresponds to a thin vortex filament when the circumferentially averaged vortex sheet has vanishing thickness and a jump in the velocity.

Using the relationship between the flux of moment of momentum downstream the runner and the discharge, as shown in Fig. 3 for constant turbine head, we can examine the evolution at runner outlet for variable discharge. Fig. 7 shows the maps

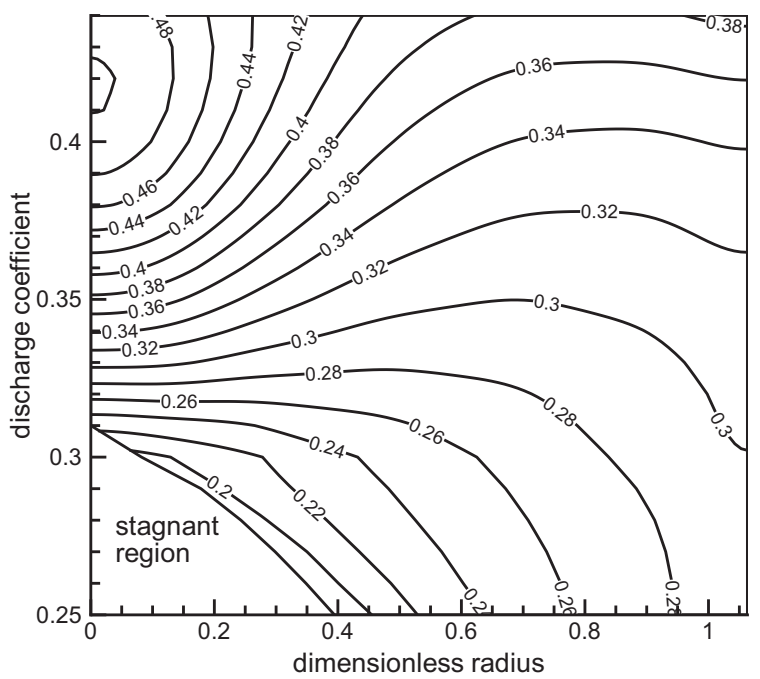

(a) Axial velocity

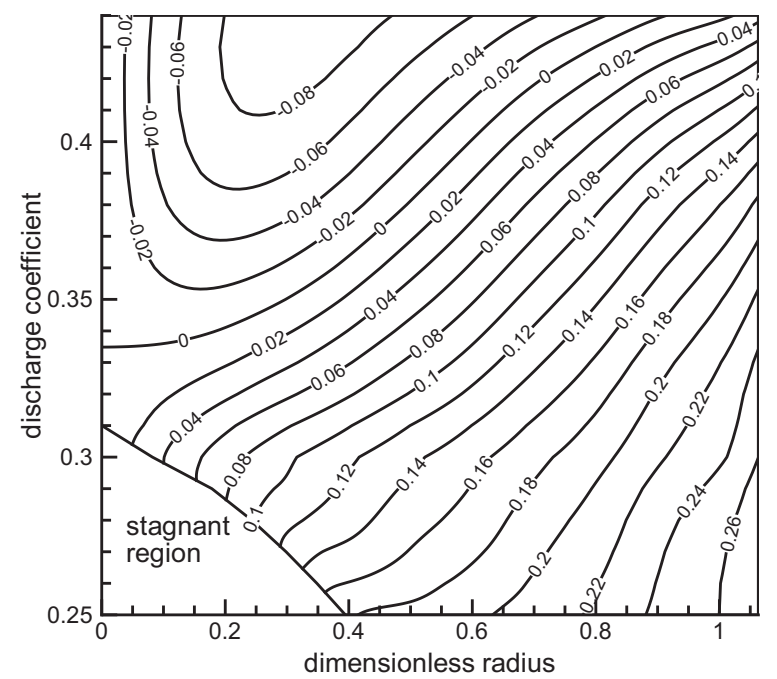

(b) Circumferential velocity

Fig. 7. Axial and circumferential velocity profiles for variable discharge coefficient $\phi=0.25 \cdots 0.44$. 
of axial and circumferential velocity components versus the radial coordinate and discharge. The radial profiles of the velocity components shown in Fig. 6 are horizontal slices of the 2D maps from Fig. 7. One can immediately identify the occurrence and development of the central stagnant region as the discharge decreases, as shown on the lower-left corner of the plots. Fig. 7(a) shows the excess in axial velocity at the axis for large discharge, and the graduate flow deceleration at the axis as the discharge decreases. However, our model predicts the development of the stagnant region before the axial velocity vanishes at the axis. Fig. 7(b) shows the development of the counter-rotation in the central region as the discharge increases, with the increase of the radius of vanishing circumferential velocity. For low discharge values the whole flow rotates in the same direction with the runner, and the circumferential velocity does not vanish at the stagnant region. In our opinion, it is this jump in both axial and circumferential velocity components at the stagnant region boundary, with a corresponding vortex sheet, which triggers the self-induced instabilities of the swirling flow and the development of the helical precessing vortex. It is clear that from the design point of view, the swirl-free velocity profile must be optimized such that the stagnant region shown in Fig. 7 be reduced and moved to lower values of the discharge.

\section{Conclusions}

We introduce a complete mathematical methodology for computing the swirling flow, i.e. radial profiles of axial and circumferential velocity components, respectively, at the runner outlet for Francis hydraulic turbines operated within the full admissible range. Since this methodology does not require the computation of the flow in the runner, it is suitable for early stages of turbine design and optimization.

Using the basic Euler equation of turbomachines, together with several considerations on the flow generated by the guide vanes of the turbine, we find a set of algebraic equations which allow the correlation between the flux of moment of momentum downstream the runner and the turbine operating regimes. As a result, given the turbine discharge and head, one can evaluate the level of swirl for the flow exiting the runner and further ingested by the draft tube.

For the fixed pitch runners, such as Francis hydraulic turbines, the swirling flow at runner outlet must satisfy a kinematic constraint usually given by the relative flow angle. However, we introduce in this paper the swirl-free velocity as a more suitable approach to describe the swirl kinematics at runner outlet. We show that the radial profile of the hypothetical swirl-free velocity at runner outlet is practically unchanged by the operating regime once three-dimensional and viscous effects of the flow in the inter-blade channels are neglected in the first approximation. The kinematic constraint on the swirl exiting the runner provides a simple relationship for computing the circumferential velocity once the radial profile of the axial velocity is known.

Several simplified assumptions are made on the swirl at runner outlet, consistent with the flow conditions in Francis turbines. We assume a steady, axisymmetric, parallel flow, of an inviscid and incompressible fluid. As a result, such swirling flow must minimize the so-called flow force, a functional introduced by Benjamin [5]. Using the kinematic constraint at runner outlet and the radial equilibrium equation, we express the flow force functional only with the axial velocity. Moreover, in agreement with the flow physics and experimental data, we modify the functional to allow the development of a central stagnant region. The radial profile of the axial velocity component is found by minimizing the flow force functional, while maintaining the overall discharge and flux of moment of momentum, resulting in a constrained variational problem. This is the core of the mathematical methodology introduced in this paper.

In order to solve numerically the variational problem we represent the unknown axial velocity as a truncated FourierBessel series. We show that by suitably choosing the first coefficient in this series the overall discharge constraint is identically satisfied. As a result, the flow force functional must be minimized with respect to the set of mode coefficients in the Fourier-Bessel series and the additional unknown for the stagnant region radius, respectively, while satisfying only the constraint for the flux of moment of momentum. The resulting numerical algorithm is robust, most likely thanks to the FourierBessel modes orthogonality.

The above mathematical methodology is validated against available experimental data for axial and circumferential velocity profiles measured at the runner outlet of a Francis model turbine for seven operating regimes. We show that our model correctly captures the swirling flow evolution with the variable operating regime of the Francis turbine. However, as expected the flow features associated with viscous effects, such as the runner hub wake, or three-dimensional effects, such as inter-blade vortices, cannot be captured within the simplified assumptions of our model.

Finally, let us summarize the main advantages and potential applications of the mathematical model introduced in this paper:

(i) The model allows the computation of the swirling flow, i.e. radial profiles of axial and circumferential velocity components, at the runner outlet of Francis turbines for any operating regime without any computation of the threedimensional flow in the turbine.

(ii) The runner blades geometry at the trailing edge is embedded in the swirl-free velocity profile. The radial profile of the swirl-free velocity has a simple shape, and a simple two-parameter representation is quite suitable for optimizing the swirling flow ingested by the turbine' s draft tube before designing the runner. This is essential for modern hydraulic turbines where the shape of the efficiency hill-chart is actually driven by the hydraulic losses in the draft tube. 
(iii) The model is intended to be used in the early design and optimization stages of a new hydraulic turbine, or for refurbishment of existing turbines, as it allows the evaluation of a large number of operating regimes and design choices with very low computational costs.

(iv) Since the model correctly captures the development of the central stagnant region when operating the turbine far from the design operating regime, one can estimate in the early design stages the boundaries of the smooth operation range.

(v) Once an optimum swirling flow configuration at runner outlet is achieved by optimizing the swirl-free velocity in conjunction with the performances of the draft tube, the runner blades geometry follows through an inverse design approach.

Due to the simplified assumptions taken into account, the present model is recommended for $a$ priori evaluation of design choices for hydraulic turbines, leaving the a posteriori evaluation to the current experimental protocols or to the full threedimensional viscous flow computations.

\section{Acknowledgements}

Prof. Romeo Susan-Resiga and Dr. Sebastian Muntean were supported by CNCSIS - UEFISCSU, Exploratory Research Project number PN II - IDEI code 799/2008. Moreover the authors wish to thanks the partners of the FLINDT Project Eureka No. 1625, Alstom Power Hydro, Electricité de France, GE Hydro, VA Tech Hydro, Voith-Siemens Hydro, PSEL Funds for Projects and Studies of the Swiss Electric Utilities, and the CTI Commission for Technology and Innovation, for their financial support.

\section{References}

[1] M. Escudier, Confined vortices in flow machinery, Annual Rev. Fluid Mech. 19 (1987) 27-52.

[2] T.C. Vu, S. Retieb, Accuracy assessment of current cfd tools to predict hydraulic turbine efficiency hill chart, in: Proceedings of the 21st IAHR Symposium on Hydraulic Machinery and Systems, volume 1, École Polytechnique Fédérale de Lausanne, Lausanne, Switzerland, 2002, pp. 193-198.

[3] F. Avellan, Flow investigation in a Francis draft tube: the FLINDT project, in: Proceedings of the 20th IAHR Symposium on Hydraulic Machinery and Systems, IAHR, Charlotte (NC), U.S.A., 2000. Paper DY-03.

[4] R. Susan-Resiga, G.D. Ciocan, I. Anton, F. Avellan, Analysis of the swirling flow downstream a Francis turbine runner, J. Fluids Eng. 128 (2006) 177-189.

[5] T.J. Benjamin, Theory of the vortex breakdown phenomenon, J. Fluid Mech. 14 (1962) 593-629.

[6] S. Mauri, J.L. Kueny, F. Avellan, Werlé-Legendre separation in a hydraulic machine draft tube, J. Fluid Eng. 126 (2004) 976-980.

[7] R. Susan-Resiga, S. Muntean, V. Hasmaţuchi, I. Anton, F. Avellan, Analysis and prevention of vortex breakdown in the simplified discharge cone of a francis turbine, J. Fluid Eng. 132 (2010) 15 pages. 051102.

[8] G.D. Ciocan, M.S. Iliescu, T.C. Vu, B. Nennemann, F. Avellan, Experimental study and numerical simulation of the FLINDT draft tube rotating vortex, J. Fluids Eng. 129 (2007) 146-158.

[9] J. Arpe, C. Nicolet, F. Avellan, Experimental evidence of hydroacoustic pressure waves in a Francis turbine elbow draft tube for low discharge conditions, J. Fluids Eng. 131 (2009) 9. Paper 081102.

[10] R. Susan-Resiga, S. Muntean, P. Stein, F. Avellan, Axisymmetric swirling flow simulation of the draft tube vortex in Francis turbines at partial discharge, Int. J. Fluid Mach. Sys. 2 (2009) 295-302.

[11] A. Escue, J. Cui, Comparison of turbulence models in simulating swirling pipe flows, Appl. Math. Model. 34 (2010) 2840-2849, doi:10.1016/ j.apm.2009.12.018

[12] R. Susan-Resiga, T.C. Vu, S. Muntean, G.D. Ciocan, B. Nennemann, Jet control of the draft tube vortex rope in francis turbines at partial discharge, in: Proceedings of the 23rd IAHR Symposium on Hydraulic Machinery and Systems, IAHR, Yokohama, Japan, 2006. Paper F192 (on CD-ROM).

[13] R.K. Zhang, J.Z. Wu, S.Y. Chen, Y.L. Wu, S.H. Liu, Characteristics and control of the draft-tube flow in part-load Francis turbine, J. Fluids Eng. 131 (2009) pp 13. Paper 021101

[14] A. Ruprecht, T. Helmrich, T. Aschenbrenner, T. Scherer, Simulation of vortex rope in a turbine draft tube, in: Proceedings of the 21st IAHR Symposium on Hydraulic Machinery and Systems, volume 1, École Polytechnique Fédérale de Lausanne, Lausanne, Switzerland, 2002, pp. 259-266.

[15] P. Stein, Numerical Simulation and Investigation of Draft Tube Vortex Flow, Ph.D. thesis, Coventry University, 2007.

[16] M.T. Bovet, Contribution a l'étude du tracé d'aubage d'une turbine a réaction du type Francis, Technical Report 9, Information Techniques Charmilles, 1963.

[17] R.W. Fox, A.T. McDonald, R.W. Van Dewoestine, Effects of swirling inlet flow on pressure recovery in conical diffusers, AIAA J. 9 (1971) $2014-2018$.

[18] I. Anton, Hydraulic Turbines, Ed. Facla, Timişoara, 1979, (in Romanian).

[19] M. Zangeneh, A compressible three-dimensional desigh method for radial and mixed flow turbomachinery blades, Int. J. Numer. Methods Fluids 13 (1991) 599-624.

[20] S. Wang, Z. Rusak, The dynamics of a swirling flow in a pipe and transition to axisymmetric vortex breakdown, J. Fluid Dyn. 340 (1997) 177-223.

[21] B. Leclaire, D. Sipp, A sensitivity study of vortex breakdown onset to upstream boundary conditions, J. Fluid Mech. 645 (2010) 81-119.

[22] IMSL MATH/LIBRARY: FORTRAN Subroutines for Mathematical Applications, Visual Numerics Inc., 1994.

[23] T. Kubota, F. Han, F. Avellan, Performance analysis of the draft tube for GAMM Francis turbine, in: E. Cabrera, V. Espert, F. Martinez (Eds.), Hydraulic Machinery and Cavitation, vol.1, Kluwer Academic Publishers, Dordrecht, The Netherlands, 1996, pp. 130-139.

[24] S.L. Bragg, W.R. Hawthorne, Some exact solutions of the flow through annular cascade actuator discs, J. Aero. Sci. 17 (1950) 243.

[25] M. Frewer, M. Oberlack, S. Guenter, Symmetry investigations on the incompressible stationary axisymmetric Euler equations with swirl, Fluid Dyn. Res. 39 (2007) 647-664.

[26] G.C. Oates, C.J. Knight, G.F. Carey, A variational formulation of the compressible throughflow problem, J. Eng. Power (1976) 1-8.

[27] J.J. Keller, W. Egli, J. Exley, Force- and loss-free transitions between flow states, J. Appl. Math. Phy. (ZAMP) 36 (1985) 854-889.

[28] M.J. Cervantes, H. Gustavsson, On the use of the Squire-Long equation to estimate radial velocities in swirling flows, J. Fluids Eng. 129 (2007) 209-217.

[29] S. Tridon, S. Barre, G.D. Ciocan, L. Tomas, Experimental analysis of the swirling flow in a francis turbine draft tube: Focus on radial velocity component determination, Euro. J. Mech. B/Fluids 29 (2010) 321-335.

[30] M. Nishi, S. Matsunaga, M. Okamoto, M. Uno, K. Nishitani, Measurement of three-dimensional periodic flow in a conical draft tube at surging condition, in: U.S. Rohatgi (Ed.), Flows in Non-Rotating Turbomachinery Components, FED, vol. 69, ASME, 1988, pp. 81-88.

[31] O. Kirschner, A. Ruprecht, Vortex rope measurement in a simplified draft tube, in: R. Susan-Resiga, S. Muntean, S. Bernad (Eds.), Proceedings 2nd IAHR International Meeting of the Workgroup on Cavitation and Dynamic Problems in Hydraulic Machinery and Systems, vol. 52, Transactions on Mechanics, Politehnica University of Timişoara of Scientific Bulletin, Romania, 2007, pp. 81-88. 
[32] S.V. Alekseenko, P.A. Kuibin, V.L. Okulov, S.I. Shtork, Helical vortices in swirl flow, J. Fluid Mech. 382 (1999) 195-243.

[33] F. Bowman, Introduction to Bessel Functions, Dover Publications Inc.,, New York, 1958.

[34] K. Schittkowski, NLPQL: A FORTRAN subroutine solving constrained nonlinear programming problems, Ann. Oper. Res. 5 (1986) $485-500$.

[35] R.E. Piessens, E. deDoncker Kapenga, C.W. Uberhuber, D.K. Kahaner, QUADPACK, Springer-Verlag, New York, 1983.

[36] D.E. Müller, A method for solving algebraic equations using an automatic computer, Math. Tables Aids Comput. 10 (1956) $208-215$.

[37] G.D. Ciocan, F. Avellan, J.L. Kueny, Optical measurement techniques for experimental analysis of hydraulic turbines rotor-stator interaction in: Proceedings of the ASME 2000 Fluids Engineering Division Summer Meeting, ASME, Boston (MA), U.S.A., 2000, (Paper FEDSM2000-11056).

[38] P.A. Kuibin, V.L. Okulov, R. Susan-Resiga, S. Muntean, Validation of analytical formulae for predicting the vortex rope rotating frequency, in: Proceedings of the 25th IAHR Symposium on Hydraulic Machinery and Systems, vol. 12, Earth and Environmental Science of Institute of Physics Conference Series, IoP, <http://iopscience.iop.org/1755-1315/12/1/012051>, 2010, Paper 012051 (10 pp). 